Canadian

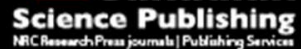

Canadian Journal of Physiology and Pharmacology Revue canadienne de physiologie et pharmacologie

\title{
Antibiotic drug piperacillin induces neuron cell death through mitochondrial dysfunction and oxidative damage
}

\begin{tabular}{|r|l|}
\hline Journal: & Canadian Journal of Physiology and Pharmacology \\
\hline Manuscript ID & cjpp-2016-0679.R2 \\
\hline Danuscript Type: & Article \\
\hline Complete List of Authors: & $\begin{array}{l}\text { Jiang, Shan; Nanning No.2 People's Hospital, Department of Neurology; } \\
\text { Li, Tong ; Nanning No.2 People's Hospital, Department of Neurology } \\
\text { Zhou, Xiao; The Affiliated Cancer Hospital of Guangxi Medical University, } \\
\text { Division of Breast Surgery } \\
\text { Qin, Wenjun; Nanning No.2 People's Hospital, Department of Neurology } \\
\text { Wang, Zijun; Nanning No.2 People's Hospital, Department of Neurology } \\
\text { Liao, Yi; Nanning No.2 People's Hospital, Department of Pharmacy }\end{array}$ \\
\hline $\begin{array}{r}\text { Is the invited manuscript for } \\
\text { consideration in a Special } \\
\text { Issue?: }\end{array}$ & N/A \\
\hline Keyword: & antibiotics, piperacillin, mitochondria, ROS, neuron disease \\
\hline \multicolumn{2}{|c|}{} \\
\hline
\end{tabular}




\title{
Antibiotic drug piperacillin induces neuron cell death through mitochondrial dysfunction and oxidative damage
}

\author{
Shan Jiang ${ }^{1 *}$, Tong $\mathrm{Li}^{1 *}$, Xiao Zhou ${ }^{2}$, Wenjun Qin ${ }^{1}$, Zijun Wang ${ }^{1}$, Yi Liao ${ }^{3}$ \\ ${ }^{1}$ Department of Neurology, Nanning No.2 People's Hospital, Nanning, P.R. China \\ ${ }^{2}$ Division of Breast Surgery, The Affiliated Cancer Hospital of Guangxi Medical University, \\ Nanning, P.R. China \\ ${ }^{3}$ Department of Pharmacy, Nanning No.2 People's Hospital, Nanning, P.R. China
}

Corresponding author: Shan Jiang, Department of Neurology, Nanning No.2 People's

Hospital, 13 Dancun Rd, Jiangnan, Nanning, Guangxi, P.R. China, 530031;

*These authors have contributed equally to this work.

Email address: jiangshan929@163.com 


\begin{abstract}
Although nerve damage/toxicity has been shown to be one of the side effects in patients given prolonged antibiotic treatment, the mechanisms of the action of antibiotics on neuron cells are not clear. In this work, we investigated the toxicity of piperacillin (an antibiotic that can penetrate blood-brain barrier) on neuron cells and its underlying mechanisms. We show that clinically relevant doses of piperacillin induce apoptosis in SH-SY5Y and human primary neuron cells through activating caspase 3 activity and decreasing Mcl-1 and Bcl-2 levels. In addition, piperacillin causes mitochondrial dysfunction in neuron cells as shown by the reduction of mitochondrial respiration, membrane potential, and ATP production. We further demonstrate that piperacillin increases accumulation of mitochondrial superoxide and ROS, suggesting the oxidative stress in neuron cells. Consistently, oxidative damage to DNA, proteins, and membrane lipids are observed in neuron cells exposed to piperacillin. The deleterious effects of piperacillin are abolished in neuron cells by antioxidant N-acetyl-Lcysteine (NAC), further confirming that piperacillin causes neuron cell death through inducing mitochondrial dysfunction and oxidative damage. Our work demonstrates the role of piperacillin in inducing oxidative damage in neuron cells and also provides a therapeutic strategy to prevent the side effects of antibiotic treatment.
\end{abstract}

Key words: antibiotics, piperacillin, mitochondria, ROS, neuron disease 


\section{Introduction}

Antibiotics have led to a significant reduction in morbidity and mortality associated with bacterial infections. $\beta$-lactam antibiotics are the most commonly used in clinical practice due to their strong antibacterial effects and broad antimicrobial spectra (Elander 2003; Holten and Onusko 2000). Despite the great benefits, the common side effects of antibiotic include tendinopathy, nephrotoxicity and ototoxicity (Brummett and Fox 1989; Khaliq and Zhanel 2003; Mingeot-Leclercq and Tulkens 1999). The modes of action and targets of antibiotics in bacteria have been well investigated, such as inhibition of bacterial cell wall synthesis and prevention DNA replication (Drlica and Zhao 1997; Lamb et al. 2015). Recent studies highlight the significant roles of majority of antibiotics, regardless of their action modes, on mitochondrial inhibition and the production of excessive lethal reactive oxygen species (ROS) in bacterial (Foti et al. 2012; Wang and Zhao 2009; Wang et al. 2010). Similarly, antibiotics have been shown to inhibit mitochondrial functions and induce oxidative stress in mammalian cells (Kalghatgi et al. 2013; Lamb et al. 2015).

Mitochondrial damage and oxidative stress largely contribute to the pathogenesis and progression of many neurodegeneration diseases, including brain aging and Alzheimer disease (Brewer 2000). It has been shown that mitochondrial damage leads to ATP depletion, loss of respiratory capacity, oxidative stress induced by ROS accumulation, induction of mitochondrial-specific cell death (Braun 2012; Krantic et al. 2007). We hypothesized that those antibiotics that target mitochondria in bacteria induce mitochondrial dysfunction, leading to oxidative damage mediated death in neuron cells. Various studies have demonstrated the ability of antibiotics in inducing human tumor cell death (Li et al. 2017; Song et al. 2016; Zhao et al. 2016). However, the effects of antibiotics on human normal neuron cells remain unclear. 
In this work, we focused on determining whether an antibiotic piperacillin affect human neuron cell mitochondrial functions and survival. Piperacillin was selected for this study due to its ability to penetrate the blood-brain barrier. We demonstrated that piperacillin caused mitochondrial dysfunction, ROS accumulation and death in SH-SY5Y as well as primary human neuron cells. We further showed that these deleterious effects could be reversed by antioxidant. Our work provide the fundamental evidence on the inhibitory effects of piperacillin on neuron cells and therapeutic strategy to alleviate the adverse side effects of long-term antibiotic treatment.

\section{Materials and methods}

\section{Cell culture and drugs}

Human SH-SY5Y cell line was purchased from American Type Culture Collection (ATCC) and maintained as the manufacture's instructions. SH-SY5Y cells were grown in 1: 1 mixture of Eagle's Minimum Essential Medium (ATCC, US) and F12 medium supplemented with 10\% fetal bovine serum (FBS, Hyclone, UK) and penicillin/streptomycin (Sigma, US). Human primary neuron cells (ScienCell, US) were maintained in complete Neuronal Medium (ScienCell, US). Piperacillin sodium salt and N-Acetyl-L-cysteine (NAC) were purchased from Sigma and reconstituted in $\mathrm{H}_{2} \mathrm{O}$.

\section{Apoptosis assay}

Cells were treated with piperacillin, NAC or both for 72 hours. Cells were then harvested by trypsinization and resuspended for Annexin V-FITC and Propidium Iodide (PI, BD Pharmingen, US) staining according to manufacture's recommendations. Labelled cells were processed at the flow cytometry on a Beckman Coulter FACS Calibur machine. Analysis was performed using FlowJo version 7.7.1 (TreeStar, Ashland, OR). 


\section{Western blot analyses}

Whole-cell proteins were prepared using 1X RIPA lysis buffer (Invitrogen, US) supplemented with freshly prepared protease and phosphatase inhibitors (Invitrogen, US). Equal amount of protein extracts were resolved by gel electrophoresis on SDSpolyacrylamide and then processed for Western blot analyses. Imaging was performed using GBOX system. The antibodies recognizing caspase3, Mcl-1, Bcl-2, $\gamma$-H2AX (serine 139) and $\beta$-actin (Cell Signaling Technology, US) were used. All proteins were normalized to loading control.

\section{Measurement of mitochondrial activities}

Cells were treated with drug for $24 \mathrm{~h}$ prior to measuring mitochondrial activities. Oxygen Consumption Rate (OCR) was measured at $37^{0} \mathrm{C}$ using a Seahorse XF24 extracellular flux analyser (Seahorse Bioscience, US) according to Seahorse Bioscience protocols. Briefly, cells were seeded at 50,000 cells/well on XF24 tissue culture plates and treated with drugs for $24 \mathrm{~h}$. Plate was transferred to XF24 analyzer and OCR were measured under basal conditions, in the presence of oligomycin $(0.5 \mu \mathrm{M})$ or antimycin $\mathrm{A}(0.25 \mu \mathrm{M})$, or in the presence of Carbonyl cyanide-p-trifluoromethoxyphenylhydrazone (FCCP, $0.5 \mu \mathrm{M}$ ) to assess maximal mitochondrial respiration. Mitochondrial potential was labelled by using 5,5',6,6'-tetrachloro1,1',3,3'-tetraethyl benzimidazolylcarbocyanine iodide (JC-1, Invitrogen) according to manufacture's instructions. Labelled cells were resuspended in PBS prior to being read on a Beckman Coulter FACS Calibur machine. ATP levels were measured by ATPlite Luminiescent Assay kit (Perkin Elmer, US) according to the manufacturer's protocol.

\section{Measurement of Reactive Oxygen Species (ROS) and mitochondrial superoxide}


Cells were treated with drug for $24 \mathrm{~h}$ prior to measuring ROS and mitochondrial superoxide. To detect ROS or mitochondrial superoxide, cells were incubated with $10 \mu \mathrm{M}$ CM$\mathrm{H}_{2}$ DCFDA (Life Technologies, US) at $37^{\circ} \mathrm{C}$ for $1 \mathrm{~h}$ or MitoSox Red at $37^{\circ} \mathrm{C}$ for $20 \mathrm{~min}$, respectively. The absorbance at ex/em of $495 / 525 \mathrm{~nm}$ or $510 / 580 \mathrm{~nm}$ were measured using Spectramax M5 microplate reader (Molecular Devices).

\section{Measurement of oxidative DNA, protein, and lipid Damage}

Cells were treated with drug for $24 \mathrm{~h}$ prior to measuring oxidative damage. To measure oxidative DNA damage, DNA was extracted using the DNEasy Mini Kit (Qiagen, US). 8hydroxy-2'-deoxyguanosine (8-OHdG) levels were quantified using the OxiSelect Oxidative DNA Damage ELISA Kit (Cell Biolabs), following the manufacturer's protocol. Absorbance at $450 \mathrm{~nm}$ was read on a Spectramax M5 Microplate. Protein carbonylation was measured using the Protein Carbonyl ELISA Kit (Enzo LifeSciences). Briefly, proteins extracted from samples and standards proteins were derivative with dinitrophenylhydrazine (DNP) and ELISA was carried out to measure protein carbonyl content according to manufacture's protocol. Lipid peroxidation was measured by using the Lipid Peroxidation MDA Assay Kit (Abcam, US) according to the manufacture's instructions.

\section{Statistical analyses}

The data are expressed as mean and standard deviation. Statistical analyses were performed by unpaired Student's t test. Values were considered statistically significant at $p<0.05$.

\section{Results}

\section{Piperacillin significantly induces neuron cell death.}

SH-SY5Y can grow continuously as undifferentiated cells that have a neuroblast-like morphology and is one of the most commonly used models to study neuronal cells (Gordon et 
al. 2013). We first investigated whether clinically relevant doses of piperacillin affect survival in neuron cells using SH-SY5Y as well as human primary neurons. The clinical relevant does here are defined by the peak plasma drug concentrations after distribution has reached equilibrium (Levison and Levison 2009). We found that piperacillin dosedependently induced apoptosis in SH-SY5Y and human primary neuron cells as assessed by Annexin V and PI staining (Fig. 1A and B). Hoechst staining also showed the increased number of neuron cells with condensed chromatin after piperacillin treatment (supplementary Fig. S1). We further found that piperacillin induced $\sim 10 \%, \sim 40 \%, \sim 55 \%$ apoptosis after $24 \mathrm{~h}$, 48h, and $72 \mathrm{~h}$ treatment, respectively (Fig. 1C). This suggests that piperacillin induces apoptosis at late time stage such as 48 and $72 \mathrm{~h}$. Consistent with the Annexin V and PI staining results, we also observed the increased levels of cleaved caspase- 3 and decreased levels of essential anti-apoptotic molecules, such as Bcl-2 and Mcl-1, in SH-SY5Y and human primary neuron cells exposed to piperacillin (Fig.1C). These results clearly demonstrate the pro-apoptotic effects of antibiotic piperacillin in human neuron cells.

\section{Piperacillin significantly induces mitochondrial dysfunction and oxidative stress in neuron cells.}

Antibiotics from $\beta$-lactam class have been shown to inhibit mitochondrial biogenesis and induces oxidative stress in mammalian cells (Kalghatgi et al. 2013; Lamb et al. 2015). We therefore examined whether piperacillin affects mitochondrial functions in neuron cells by measuring mitochondrial OCR, membrane potential and ATP levels. We found that piperacillin significantly decreases basal and maximal OCR (indicative of reserved respiratory capacity) in SH-SY5Y and primary neuron cells in a dose-dependent manner (Fig. $2 \mathrm{~A}$ and $\mathrm{B})$. In addition, decreased mitochondrial membrane potential and ATP production were observed in neuron cells exposed to piperacillin (Fig. 2C and D). Superoxide is a 
precursor to many forms of ROS and generated by leakage of electrons from the mitochondrial electron transport chain (Turrens 2003). Given the inhibitory effects of piperacillin on mitochondrial respiration, we tested the mitochondrial superoxide and intracellular ROS levels in neuron cells. We found that piperacillin dose-dependently increased mitochondrial superoxide and intracellular ROS levels in SH-SY5Y and primary neuron cells (Fig. 2E and F), demonstrating that piperacillin induces oxidative stress in neuron cells.

\section{Piperacillin causes oxidative damage to neuron cells.}

We next determined whether oxidative stress induced by piperacillin leads to oxidative DNA damage in neuron cells by analyzing the levels of $\gamma-\mathrm{H} 2 \mathrm{AX}$ (a core histone protein in response to DNA damage) and 8-OHdG (an oxidized DNA byproduct). Western blot analyses showed that $\gamma-\mathrm{H} 2 \mathrm{AX}$ levels were significantly increased in neuron cells treated with piperacillin (Fig. 3A and supplementary Fig. S2). Similarly, the increased levels of 8-OHdG were observed in neuron cells after piperacillin treatment (Fig. 3B), confirming that piperacillin induces oxidative DNA damage. We further found that piperacillin induced protein and lipid damage as shown by the increased levels of protein carbonyls (a modification of proteins resulting from oxidative damage) and malondialdehyde (MDA, an end product of lipid peroxidation) in neuron cells treated with piperacillin (Fig. $3 \mathrm{C}$ and D). Taken together, piperacillin induces oxidative DNA, protein and lipid damage in neuron cells.

\section{NAC rescues piperacillin-induced mitochondrial dysfunctions, oxidative damage and cell death in neuron cells.}

We then investigated whether piperacillin-induced these deleterious effects can be rescued by antioxidant. NAC is an antioxidant and commonly used to buffer extraneous ROS in mammalian cells (Aruoma et al. 1989). SH-SY5Y and primary neuron cells were pretreated 
with NAC, followed by piperacillin treatment. We found that NAC pretreatment restored mitochondrial respiration and membrane potential, and ATP production to similar levels observed in control cells (Fig. 4). NAC pretreatment also abolished piperacillin-induced ROS levels (Fig. 5A). NAC pretreatment also reversed the effects of piperacillin in increasing levels of $\gamma$-H2AX, 8-OHdG, protein carbonyls and MDA (Fig. 5B to E and supplementary Fig. S3). Importantly, piperacillin is ineffective in inducing neuron cell apoptosis in the presence of NAC (Fig. 5F). These data indicate that oxidative damage induction is required for the action of piperacillin in neuron cells.

\section{Discussion}

It is known that antibiotics can cause adverse effects in central nervous system, such as depression and headache (Munjampalli and Davis 2016). However, the mechanisms of antibiotics on how they interact with neuron cells are largely unknown. Better understanding of the mechanisms of action of antibiotics in neuron cells, particularly for those that can penetrate blood brain-barrier, is important to accomplish a clear safety profile for antibiotics and combat the side effects of prolonged treatment. Piperacillin is a broad-spectrum $\beta$-lactam antibiotic and commonly used in combination with tazobactam for hospital-acquired pneumonia (Yim et al. 2016). In this work, we systematically investigated the toxicity of piperacillin in neuron cells and its underlying mechanisms. The cultured neuron cells SHSY5Y we selected for demonstrating the biological effects of piperacillin is a common model to study neuronal cells (Gordon et al. 2013). Apart from neuron cell line, human primary neuron cells were also applied to further confirm the effects of piperacillin. We are the first to show the significant mitochondrial dysfunction and oxidative damage by piperacillin treatment, leading to neuron cell death. 
The pro-apoptotic effect of antibiotics has been demonstrated in mammalian cells, particularly in various types of human tumour cells (Li et al. 2015; Yadav et al. 2015; Yu et al. 2016). It has been suggested that human tumour cells are more sensitive to antibiotics compared to their normal counterparts in aspects of inducing apoptosis and inhibiting proliferation (Skrtic et al. 2011; Yu et al. 2016). In agreement with the previous reports, we found that piperacillin significantly induced apoptosis in neuron cells, accompanied by a profound increase in cleaved caspase 3 and reduction in Mcl-1 and Bcl-2 levels (Fig.1 and supplementary Fig. S1). Our work adds neuron cells to the growing list of antibiotic-targeted mammalian cells and supports the previous work that antibiotics at clinically relevant doses are toxic to normal human cells.

Mechanistically, piperacillin targets mitochondria in neuron cells as shown by the decreased mitochondrial respiration, membrane potential and ATP production (Fig. 2A to D). The action of piperacillin on mitochondrial in neuron cells is similar to the action of levofloxacin in breast cancer cells and tigecycline in leukaemia cells (Skrtic et al. 2011; Yu et al. 2016). The common feature of these antibiotics is that they also target mitochondria and mitochondrial components in bacteria and induce oxidative damage (Lamb et al. 2015; Pochini et al. 2008). We further found that piperacillin caused accumulation of mitochondrial superoxide and intracellular ROS (Fig. 2E and F), and damage in DNA, protein and lipids (Fig. 3) as a consequence of oxidative stress in neuron cells. Several literatures have reported piperacillin-induced neurotoxic effects, such as encephalopathy characterized by dysarthria, tremor, behavioural changes, progressive confusion and tardive seizures (Grill and Maganti 2011; Huang et al. 2009; Lin et al. 2007). This phenomena might correlate with the abilities of piperacillin in inducing mitochondrial dysfunctions and oxidative damage in neuron cells. It is interesting that some beta-lactam antibiotic (eg, ceftriaxone) have been demonstrated to 
induce neuroprotection of diverse neurological diseases via increasing glutamate transporter expression or decreasing anti-inflammation response (Rothstein et al. 2005; Wei et al. 2012). Although piperacillin is a B-lactam type antibiotics, little is known on the possible neuroprotective effects of piperacillin. In contrast, literatures and our work demonstrates the neurotoxic effects of piperacillin and its deleterious effects on neuron cell mitochondria (Grill and Maganti 2011; Huang et al. 2009; Lin et al. 2007). Besides piperacillin, other antibiotics including levofloxacin, doxycycline and tigecycline, have been reported to acts on human cells through inducing mitochondrial dysfunction and ROS overproduction (Li et al. 2017; Song et al. 2016; Zhao et al. 2016). These suggest that the actions of antibiotics in bacteria are similar in mammalian cells.

The identification of mitochondrial dysfunction and oxidative damage provides fundamentals for understanding the action of piperacillin or other similar antibiotics in neuron cells and developing therapeutic strategy to alleviate side effects by prolonged antibiotic treatment. We show that by co-treatment with antioxidant NAC, mitochondrial dysfunctions induced by piperacillin are abolished (Fig. 4). In addition, NAC abolishes the effects of piperacillin in inducing oxidative damage and cell death (Fig. 5). These demonstrate that co-administrating antioxidants and piperacillin could be a simple treatment strategy to prevent cellular mitochondrial dysfunction and oxidative damage induced by piperacillin in patients.

In conclusion, our work show that antibiotic piperacillin causes mitochondrial dysfunction and oxidative damage in neuron cells, leading to cell death. The deleterious effects of piperacillin can be significantly alleviated by antioxidant. Our work highlights the role of oxidative damage induced by antibiotics in neuron cell death and provides therapeutic strategy to mitigate antibiotic-induced oxidative damage.

\section{Conflict of interest}


All authors declare no conflict of interest.

\section{Acknowledgement}

This work was supported by research grants provided by Science and Technology Department of Guangxi Province (ZC20133006) and Science and Technology Bureau of Nanning (No. 20120316).

\section{References}

Aruoma, O.I., Halliwell, B., Hoey, B.M., and Butler, J. 1989. The antioxidant action of Nacetylcysteine: its reaction with hydrogen peroxide, hydroxyl radical, superoxide, and hypochlorous acid. Free Radic. Biol. Med. 6(6): 593-597.

Braun, R.J. 2012. Mitochondrion-mediated cell death: dissecting yeast apoptosis for a better understanding of neurodegeneration. Front. Oncol. 2: 182. doi: 10.3389/fonc.2012.00182.

Brewer, G.J. 2000. Neuronal plasticity and stressor toxicity during aging. Exp. Gerontol. 35(9-10): 1165-1183.

Brummett, R.E., and Fox, K.E. 1989. Aminoglycoside-induced hearing loss in humans. Antimicrob. Agents Chemother. 33(6): 797-800.

Drlica, K., and Zhao, X. 1997. DNA gyrase, topoisomerase IV, and the 4-quinolones. Microbiol. Mol. Biol. Rev. 61(3): 377-392.

Elander, R.P. 2003. Industrial production of beta-lactam antibiotics. Appl. Microbiol. Biotechnol. 61(5-6): 385-392. doi: 10.1007/s00253-003-1274-y. Foti, J.J., Devadoss, B., Winkler, J.A., Collins, J.J., and Walker, G.C. 2012. Oxidation of the guanine nucleotide pool underlies cell death by bactericidal antibiotics. Science, 336(6079): 315-319. doi: 10.1126/science.1219192. 
Gordon, J., Amini, S., and White, M.K. 2013. General overview of neuronal cell culture. Methods Mol. Biol. 1078: 1-8. doi: 10.1007/978-1-62703-640-5_1.

Grill, M.F., and Maganti, R.K. 2011. Neurotoxic effects associated with antibiotic use: management considerations. Br. J. Clin. Pharmacol. 72(3): 381-393. doi: 10.1111/j.13652125.2011.03991.x.

Holten, K.B., and Onusko, E.M. 2000. Appropriate prescribing of oral beta-lactam antibiotics. Am. Fam. Physician, 62(3): 611-620.

Huang, W.T., Hsu, Y.J., Chu, P.L., and Lin, S.H. 2009. Neurotoxicity associated with standard doses of piperacillin in an elderly patient with renal failure. Infection, 37(4): 374376. doi: 10.1007/s15010-009-8373-3.

Kalghatgi, S., Spina, C.S., Costello, J.C., Liesa, M., Morones-Ramirez, J.R., Slomovic, S., Molina, A., Shirihai, O.S., and Collins, J.J. 2013. Bactericidal antibiotics induce mitochondrial dysfunction and oxidative damage in Mammalian cells. Sci. Transl. Med. 5(192): 192ra185. doi: 10.1126/scitranslmed.3006055.

Khaliq, Y., and Zhanel, G.G. 2003. Fluoroquinolone-associated tendinopathy: a critical review of the literature. Clin. Infect. Dis. 36(11): 1404-1410. doi: 10.1086/375078. Krantic, S., Mechawar, N., Reix, S., and Quirion, R. 2007. Apoptosis-inducing factor: a matter of neuron life and death. Prog. Neurobiol. 81(3): 179-196. doi: 10.1016/j.pneurobio.2006.12.002.

Lamb, R., Ozsvari, B., Lisanti, C.L., Tanowitz, H.B., Howell, A., Martinez-Outschoorn, U.E., Sotgia, F., and Lisanti, M.P. 2015. Antibiotics that target mitochondria effectively eradicate cancer stem cells, across multiple tumor types: treating cancer like an infectious disease. Oncotarget, 6(7): 4569-4584. doi: 10.18632/oncotarget.3174. 
Levison, M.E., and Levison, J.H. 2009. Pharmacokinetics and pharmacodynamics of antibacterial agents. Infect. Dis. Clin. North Am. 23(4): 791-815, vii. doi:

10.1016/j.idc.2009.06.008.

Li, H., Jiao, S., Li, X., Banu, H., Hamal, S., and Wang, X. 2015. Therapeutic effects of antibiotic drug tigecycline against cervical squamous cell carcinoma by inhibiting Wnt/betacatenin signaling. Biochem. Biophys. Res. Commun. 467(1): 14-20. doi:

10.1016/j.bbrc.2015.09.140.

Li, H., Jiao, S., Li, X., Banu, H., Hamal, S., and Wang, X. 2017. Therapeutic effects of antibiotic drug mefloquine against cervical cancer through impairing mitochondrial function and inhibiting mTOR pathway. Can. J. Physiol. Pharmacol. 95(1): 43-50. doi: 10.1139/cjpp2016-0124.

Lin, C.S., Cheng, C.J., Chou, C.H., and Lin, S.H. 2007. Piperacillin/tazobactam-induced seizure rapidly reversed by high flux hemodialysis in a patient on peritoneal dialysis. Am. J. Med. Sci. 333(3): 181-184. doi: 10.1097/MAJ.0b013e31803195e7.

Mingeot-Leclercq, M.P., and Tulkens, P.M. 1999. Aminoglycosides: nephrotoxicity. Antimicrob. Agents Chemother. 43(5): 1003-1012.

Munjampalli, S.K., and Davis, D.E. 2016. Medicinal-Induced Behavior Disorders. Neurol. Clin. 34(1): 133-169. doi: 10.1016/j.ncl.2015.08.006.

Pochini, L., Galluccio, M., Scumaci, D., Giangregorio, N., Tonazzi, A., Palmieri, F., and Indiveri, C. 2008. Interaction of beta-lactam antibiotics with the mitochondrial carnitine/acylcarnitine transporter. Chem. Biol. Interact. 173(3): 187-194. doi: 10.1016/j.cbi.2008.03.003.

Rothstein, J.D., Patel, S., Regan, M.R., Haenggeli, C., Huang, Y.H., Bergles, D.E., Jin, L., Dykes Hoberg, M., Vidensky, S., Chung, D.S., Toan, S.V., Bruijn, L.I., Su, Z.Z., Gupta, P., 
and Fisher, P.B. 2005. Beta-lactam antibiotics offer neuroprotection by increasing glutamate transporter expression. Nature, 433(7021): 73-77. doi: 10.1038/nature03180.

Skrtic, M., Sriskanthadevan, S., Jhas, B., Gebbia, M., Wang, X., Wang, Z., Hurren, R., Jitkova, Y., Gronda, M., Maclean, N., Lai, C.K., Eberhard, Y., Bartoszko, J., Spagnuolo, P., Rutledge, A.C., Datti, A., Ketela, T., Moffat, J., Robinson, B.H., Cameron, J.H., Wrana, J., Eaves, C.J., Minden, M.D., Wang, J.C., Dick, J.E., Humphries, K., Nislow, C., Giaever, G., and Schimmer, A.D. 2011. Inhibition of mitochondrial translation as a therapeutic strategy for human acute myeloid leukemia. Cancer Cell, 20(5): 674-688. doi:

10.1016/j.ccr.2011.10.015.

Song, M., Wu, H., Wu, S., Ge, T., Wang, G., Zhou, Y., Sheng, S., and Jiang, J. 2016.

Antibiotic drug levofloxacin inhibits proliferation and induces apoptosis of lung cancer cells through inducing mitochondrial dysfunction and oxidative damage. Biomed. Pharmacother. 84: 1137-1143. doi: 10.1016/j.biopha.2016.10.034.

Turrens, J.F. 2003. Mitochondrial formation of reactive oxygen species. J. Physiol. 552(Pt 2): 335-344. doi: 10.1113/jphysiol.2003.049478.

Wang, X., and Zhao, X. 2009. Contribution of oxidative damage to antimicrobial lethality. Antimicrob. Agents Chemother. 53(4): 1395-1402. doi: 10.1128/AAC.01087-08.

Wang, X., Zhao, X., Malik, M., and Drlica, K. 2010. Contribution of reactive oxygen species to pathways of quinolone-mediated bacterial cell death. J. Antimicrob. Chemother. 65(3): 520-524. doi: 10.1093/jac/dkp486.

Wei, J., Pan, X., Pei, Z., Wang, W., Qiu, W., Shi, Z., and Xiao, G. 2012. The beta-lactam antibiotic, ceftriaxone, provides neuroprotective potential via anti-excitotoxicity and antiinflammation response in a rat model of traumatic brain injury. J. Trauma Acute Care Surg. 73(3): 654-660. doi: 10.1097/TA.0b013e31825133c0. 
Yadav, V., Varshney, P., Sultana, S., Yadav, J., and Saini, N. 2015. Moxifloxacin and ciprofloxacin induces S-phase arrest and augments apoptotic effects of cisplatin in human pancreatic cancer cells via ERK activation. BMC Cancer, 15: 581. doi: 10.1186/s12885-015$1560-\mathrm{y}$

Yim, J., Smith, J.R., Barber, K.E., Hallesy, J.A., and Rybak, M.J. 2016. Evaluation of Pharmacodynamic Interactions Between Telavancin and Aztreonam or Piperacillin/Tazobactam Against Pseudomonas aeruginosa, Escherichia coli and MethicillinResistant Staphylococcus aureus. Infect. Dis. Ther. 5(3): 367-377. doi: 10.1007/s40121-0160121-2.

Yu, M., Li, R., and Zhang, J. 2016. Repositioning of antibiotic levofloxacin as a mitochondrial biogenesis inhibitor to target breast cancer. Biochem. Biophys. Res. Commun. doi: 10.1016/j.bbrc.2016.02.072.

Zhao, Y., Wang, X., Li, L., and Li, C. 2016. Doxycycline inhibits proliferation and induces apoptosis of both human papillomavirus positive and negative cervical cancer cell lines. Can. J. Physiol. Pharmacol. 94(5): 526-533. doi: 10.1139/cjpp-2015-0481. 


\section{Figure Legends}

Fig. 1: Piperacillin significantly induces neuron cell death. (A) Representative flow cytometry dot plots showing the percentage of Annexin V and PI staining in SH-SY5Y and human primary neuron cells exposed to $800 \mu \mathrm{M}$ piperacillin. (B) Piperacillin significantly induces death of SH-SY5Y and primary neuron cells in a dose-dependent manner. Cells were treated with piperacillin for $72 \mathrm{~h}$ prior to apoptosis measurement. (C) The effects of piperacillin on the levels of cleaved caspase-3, Mcl-1 and Bcl-2 in SH-SY5Y and primary neuron cells. Cells were treated with piperacillin for $48 \mathrm{~h}$ prior to western blot analyses. $* p<0.05$, compared to control.

Fig. 2: Piperacillin significantly induces mitochondrial dysfunction and oxidative stress in neuron cells. Piperacillin dose-dependently decreases basal OCR (A) and maximal OCR (B), mitochondrial membrane potential (C) and ATP levels (D) in SH-SY5Y and primary neuron cells. Piperacillin significantly increases mitochondrial superoxide (E) and intracellular ROS levels (F) in neuron cells in a dose-dependent manner. Cells were treated with piperacillin for $24 \mathrm{~h}$ prior to cellular mitochondrial functions analyses. ${ }^{*} p<0.05$, compared to control.

Fig. 3: Piperacillin causes oxidative damage to neuron cells. Oxidative DNA damage is shown by the increased levels of $\gamma-\mathrm{H} 2 \mathrm{AX}$ (A) and 8-OHdG (B) in SH-SY5Y and primary neuron cells exposed to piperacillin. (C) Oxidative protein damage is shown by the increased levels of protein carbonylation in neuron cells exposed to piperacillin. (D) Piperacillin significantly increases lipid peroxidation in neuron cells. $\gamma-\mathrm{H} 2 \mathrm{AX}$ is the phosphorylated form of H2AX at Serine 139. Cells were treated with piperacillin for $24 \mathrm{~h}$ prior to cellular oxidative damage analyses. ${ }^{*} p<0.05$, compared to control. 
Fig. 4: NAC rescues piperacillin-induced mitochondrial dysfunctions in neuron cells. NAC reverse the effects of piperacillin in decreasing levels of basal OCR (A), and maximal OCR (B), mitochondrial membrane potential (C) and ATP levels (D) in SH-SY5Y and primary neuron cells. Cells were incubated with $10 \mathrm{mM}$ NAC for $4 \mathrm{~h}$, followed by treatment with 800 $\mu \mathrm{M}$ piperacillin for $24 \mathrm{~h} .{ }^{*} p<0.05$, compared to piperacillin treatment alone.

Fig. 5: NAC rescues piperacillin-induced oxidative damage and cell death in neuron cells. NAC reverse the effects of piperacillin in increasing levels of cellular ORS (A), $\gamma$ H2AX (B) , 8-OHdG (C), carbonylation (D), MDA (E) and apoptosis (F) in SH-SY5Y and primary neuron cells. Cells were incubated with $10 \mathrm{mM} \mathrm{NAC} \mathrm{for} 4 \mathrm{~h}$, followed by treatment with $800 \mu \mathrm{M}$ piperacillin for 24 (metabolic and oxidative damage assays) or $72 \mathrm{~h}$ (apoptosis assay). ${ }^{*} p<0.05$, compared to piperacillin treatment alone. 
Fig 1

A SH-SY5Y
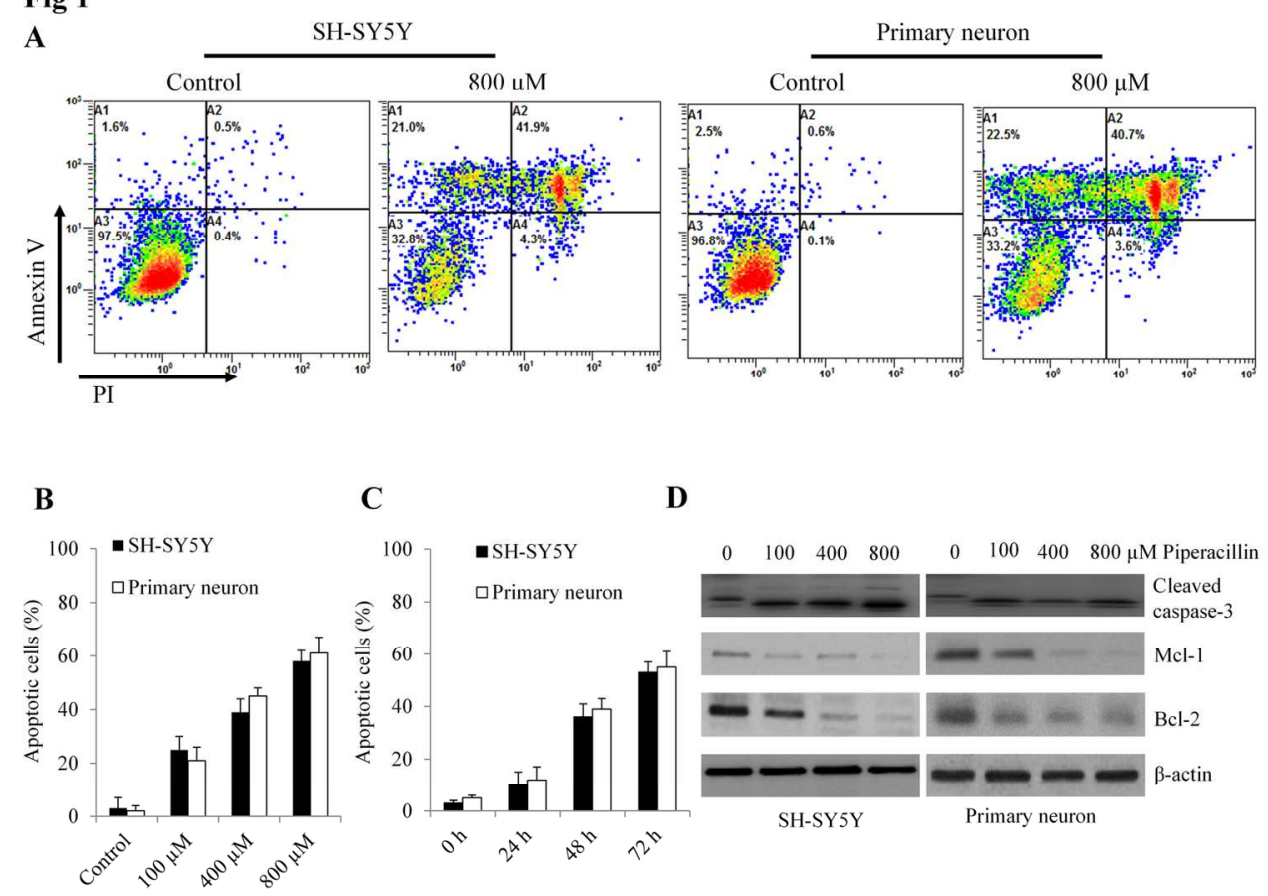

C

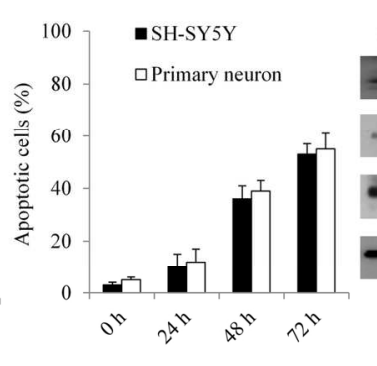

D

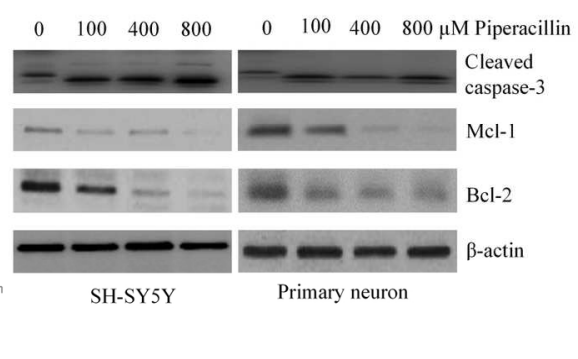

Fig. 1: Piperacillin significantly induces neuron cell death. (A) Representative flow cytometry dot plots showing the percentage of Annexin $\mathrm{V}$ and PI staining in SH-SY5Y and human primary neuron cells exposed to $800 \mu \mathrm{M}$ piperacillin. (B) Piperacillin significantly induces death of SH-SY5Y and primary neuron cells in a dose-dependent manner. Cells were treated with piperacillin for $72 \mathrm{~h}$ prior to apoptosis measurement. (C)

The effects of piperacillin on the levels of cleaved caspase-3, $\mathrm{Mcl}-1$ and $\mathrm{Bcl}-2$ in $\mathrm{SH}-\mathrm{SY} 5 \mathrm{Y}$ and primary neuron cells. Cells were treated with piperacillin for $48 \mathrm{~h}$ prior to western blot analyses. $* \mathrm{p}<0.05$, compared to control.

$154 \times 113 \mathrm{~mm}(300 \times 300$ DPI $)$ 
Fig 2
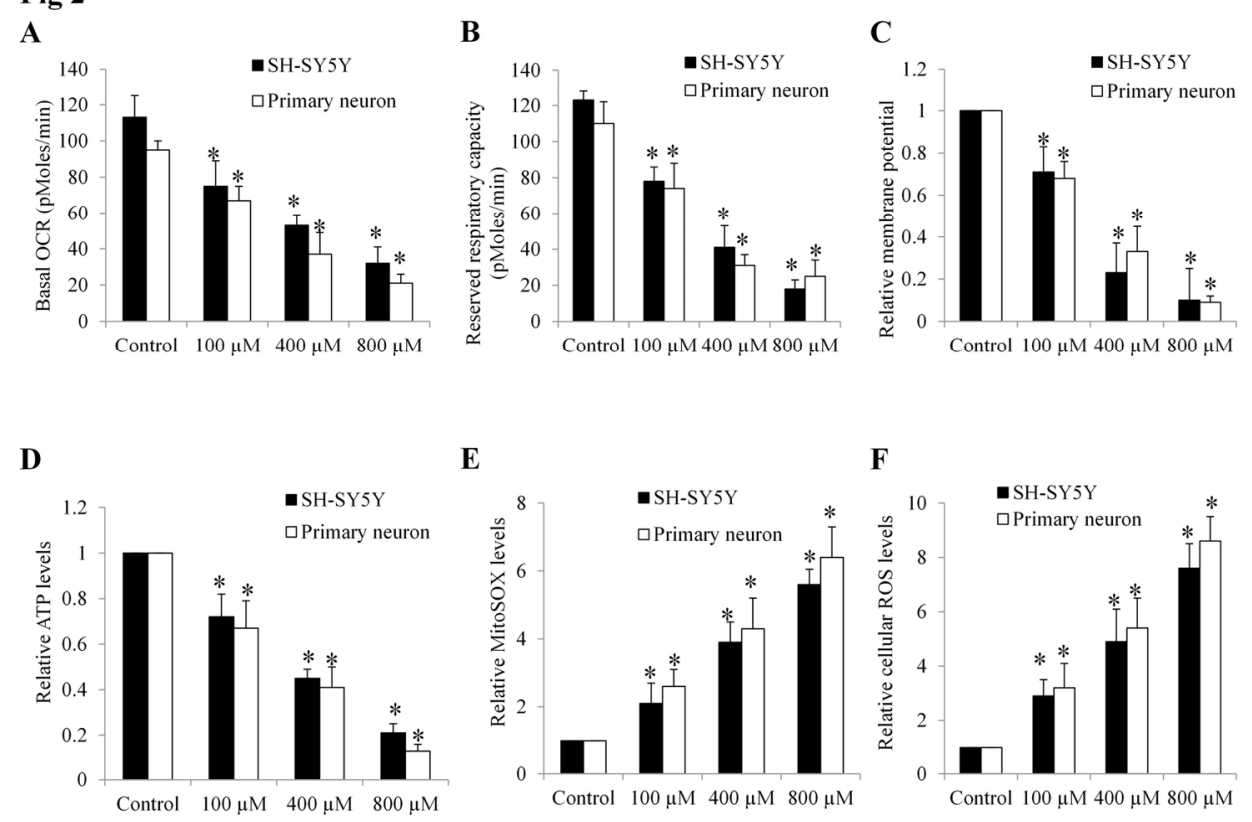

Fig. 2: Piperacillin significantly induces mitochondrial dysfunction and oxidative stress in neuron cells. Piperacillin dose-dependently decreases basal OCR (A) and maximal OCR (B), mitochondrial membrane potential (C) and ATP levels (D) in SH-SY5Y and primary neuron cells. Piperacillin significantly increases mitochondrial superoxide (E) and intracellular ROS levels (F) in neuron cells in a dose-dependent manner. Cells were treated with piperacillin for $24 \mathrm{~h}$ prior to cellular mitochondrial functions analyses. $* p<0.05$, compared to control.

$152 \times 109 \mathrm{~mm}(300 \times 300 \mathrm{DPI})$ 
Fig 3

A

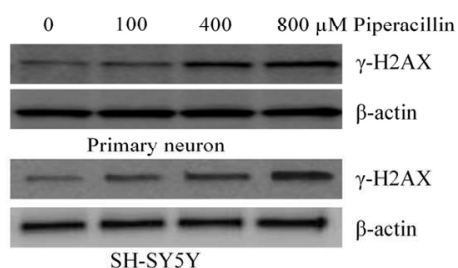

C

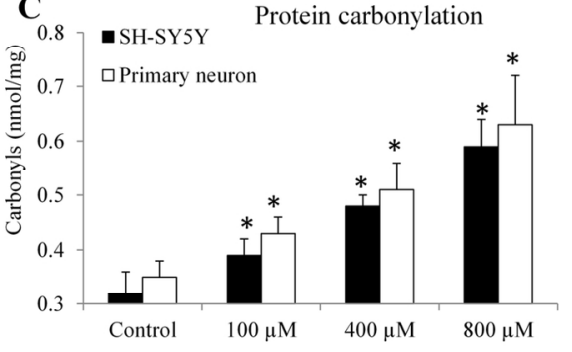

B

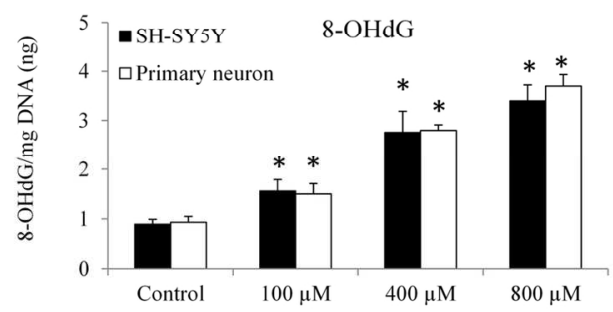

D

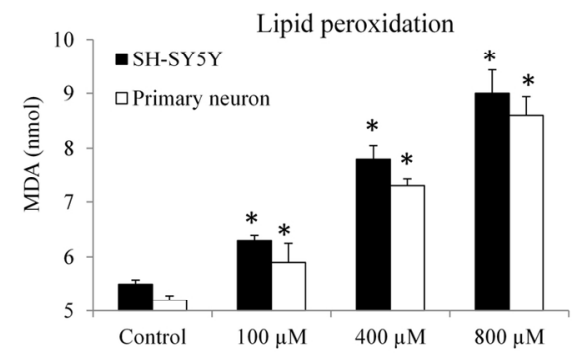

Fig. 3: Piperacillin causes oxidative damage to neuron cells. Oxidative DNA damage is shown by the increased levels of $\mathrm{Y}-\mathrm{H} 2 \mathrm{AX}(\mathrm{A})$ and 8-OHdG (B) in SH-SY5Y and primary neuron cells exposed to piperacillin. (C) Oxidative protein damage is shown by the increased levels of protein carbonylation in neuron cells exposed to piperacillin. (D) Piperacillin significantly increases lipid peroxidation in neuron cells. $\mathrm{Y}-\mathrm{H} 2 \mathrm{AX}$ is the phosphorylated form of H2AX at Serine 139. Cells were treated with piperacillin for $24 \mathrm{~h}$ prior to cellular oxidative damage analyses. $* p<0.05$, compared to control.

$$
139 \times 92 \mathrm{~mm}(300 \times 300 \mathrm{DPI})
$$


Fig 4

A

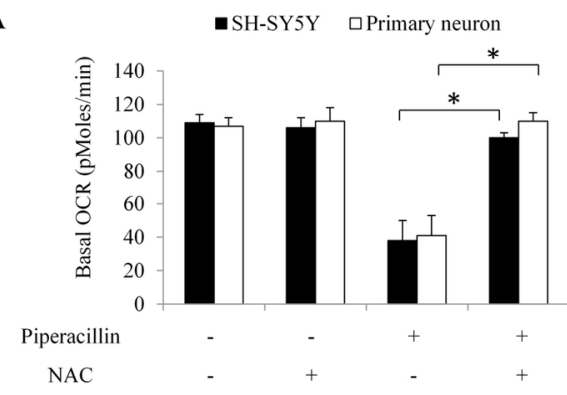

C

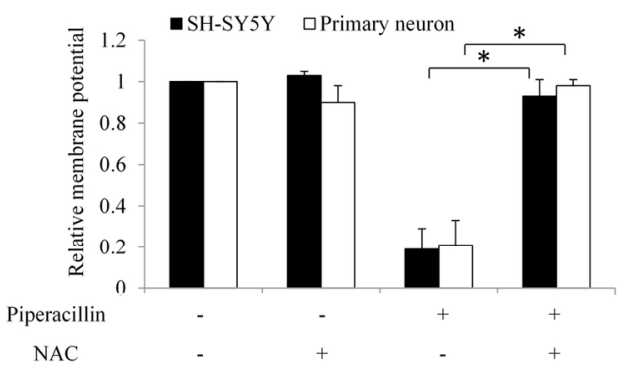

B

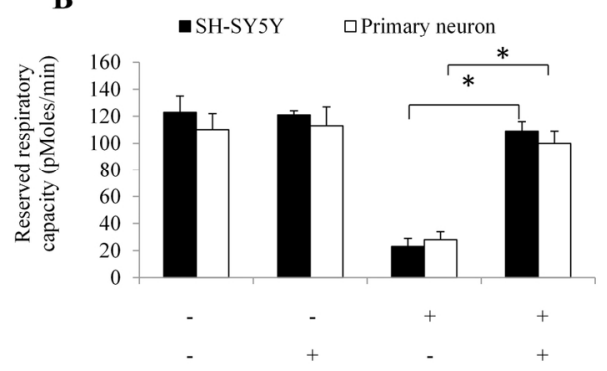

D

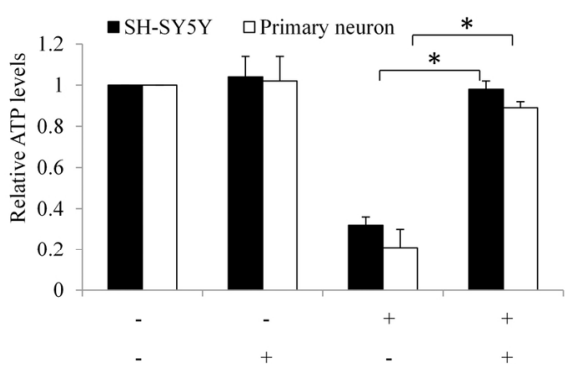

Fig. 4: NAC rescues piperacillin-induced mitochondrial dysfunctions in neuron cells. NAC reverse the effects of piperacillin in decreasing levels of basal OCR (A), and maximal OCR (B), mitochondrial membrane potential (C) and ATP levels (D) in SH-SY5Y and primary neuron cells. Cells were incubated with 10 mM NAC for $4 \mathrm{~h}$, followed by treatment with $800 \mu \mathrm{M}$ piperacillin for $24 \mathrm{~h}$. ${ }^{*} \mathrm{p}<0.05$, compared to piperacillin treatment alone.

$152 \times 109 \mathrm{~mm}(300 \times 300 \mathrm{DPI})$ 

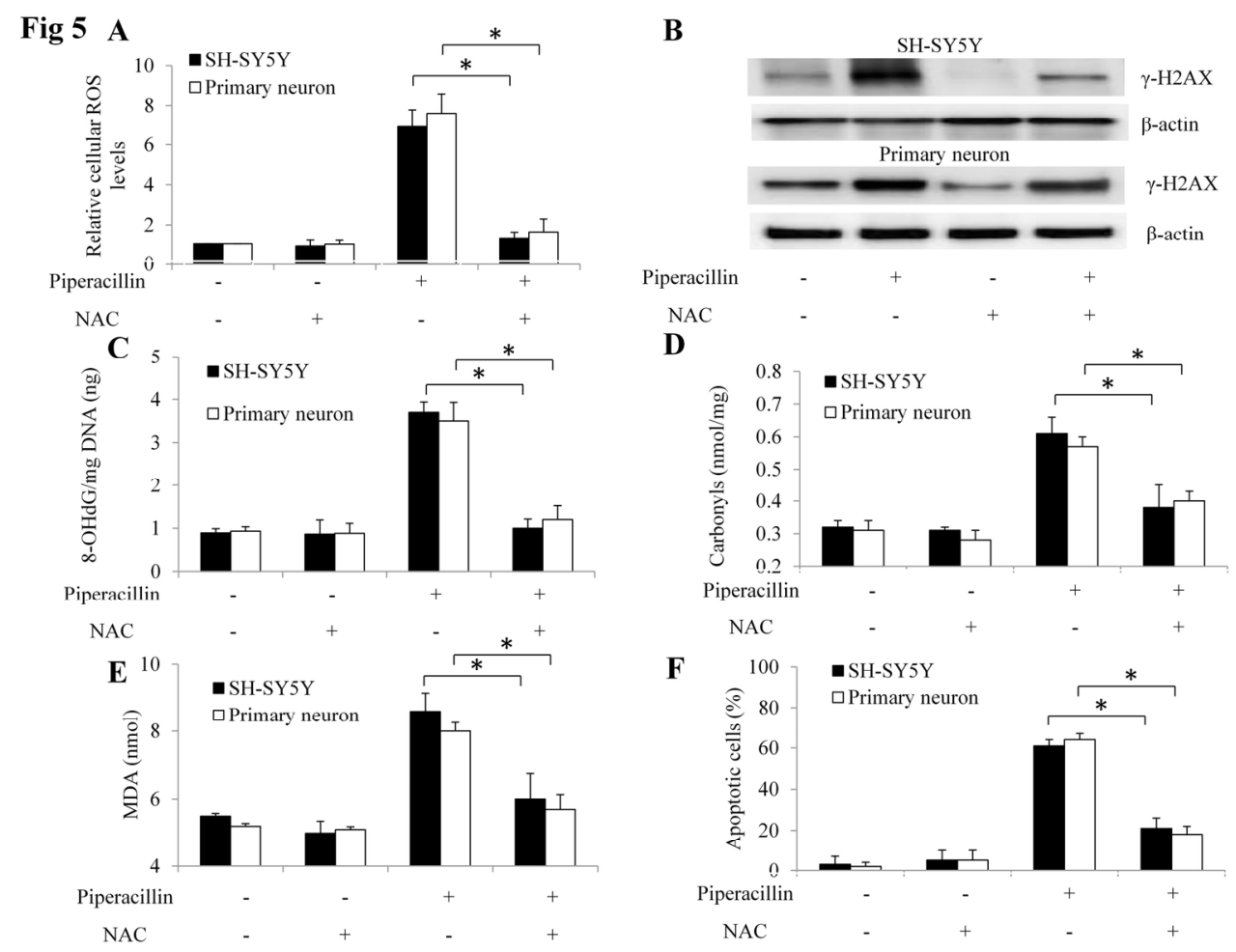

Fig. 5: NAC rescues piperacillin-induced oxidative damage and cell death in neuron cells. NAC reverse the effects of piperacillin in increasing levels of cellular ORS (A), $\mathrm{Y}$-H2AX (B), 8-OHdG (C), carbonylation (D), MDA (E) and apoptosis (F) in SH-SY5Y and primary neuron cells. Cells were incubated with $10 \mathrm{mM}$ NAC for 4 $h$, followed by treatment with $800 \mu \mathrm{M}$ piperacillin for 24 (metabolic and oxidative damage assays) or $72 \mathrm{~h}$ (apoptosis assay). ${ }^{*} p<0.05$, compared to piperacillin treatment alone.

$161 \times 123 \mathrm{~mm}(300 \times 300 \mathrm{DPI})$ 


\section{Antibiotic drug piperacillin induces neuron cell death through mitochondrial dysfunction and oxidative damage}

Shan Jiang ${ }^{1 *}$, Tong $\mathrm{Li}^{1 *}$, Xiao Zhou ${ }^{2}$, Wenjun Qin ${ }^{1}$, Zijun Wang ${ }^{1}$, Yi Liao ${ }^{3}$

\section{Materials and methods}

\section{Immunofluorescence staining}

Cells were treated with piperacillin $(800 \mu \mathrm{M})$ for $48 \mathrm{~h}$ prior to Hoechst dye 33258 (Invitrogen,

US) strictly according to manufacturer's instructions.

\section{Supplementary Fig. S1}

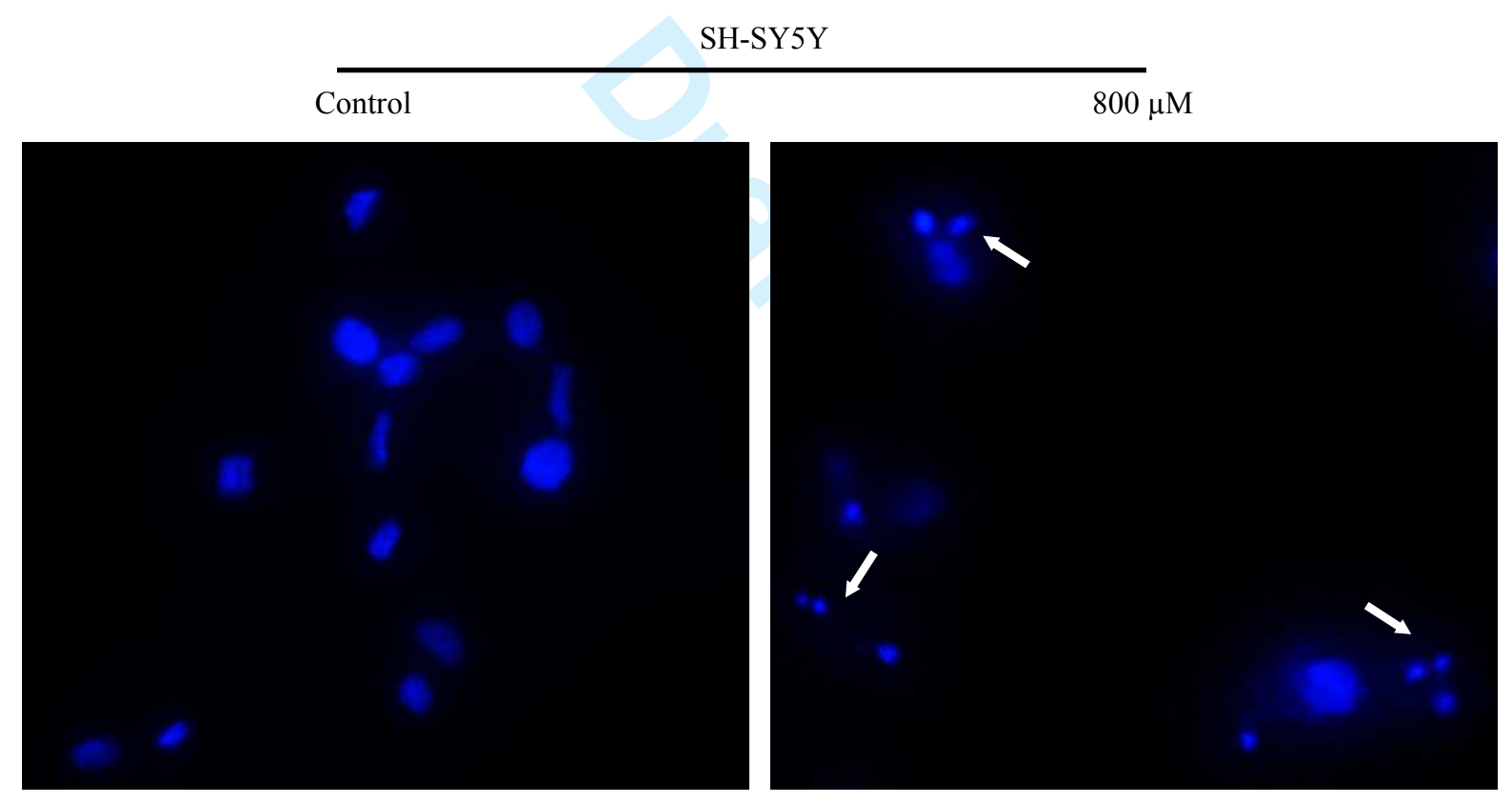

Supplementary Fig. S1. Representative fluorescence images of SH-SY5Y cells with Hoechst dye 33258 staining. Control cells exhibited diffused staining of the chromatin while condensed chromatin and shrunken nucleus (indicated by arrows) were observed in cells treated with control or $800 \mu \mathrm{M}$ piperacillin for $48 \mathrm{~h}$. 


\section{Supplementary Fig. S2}

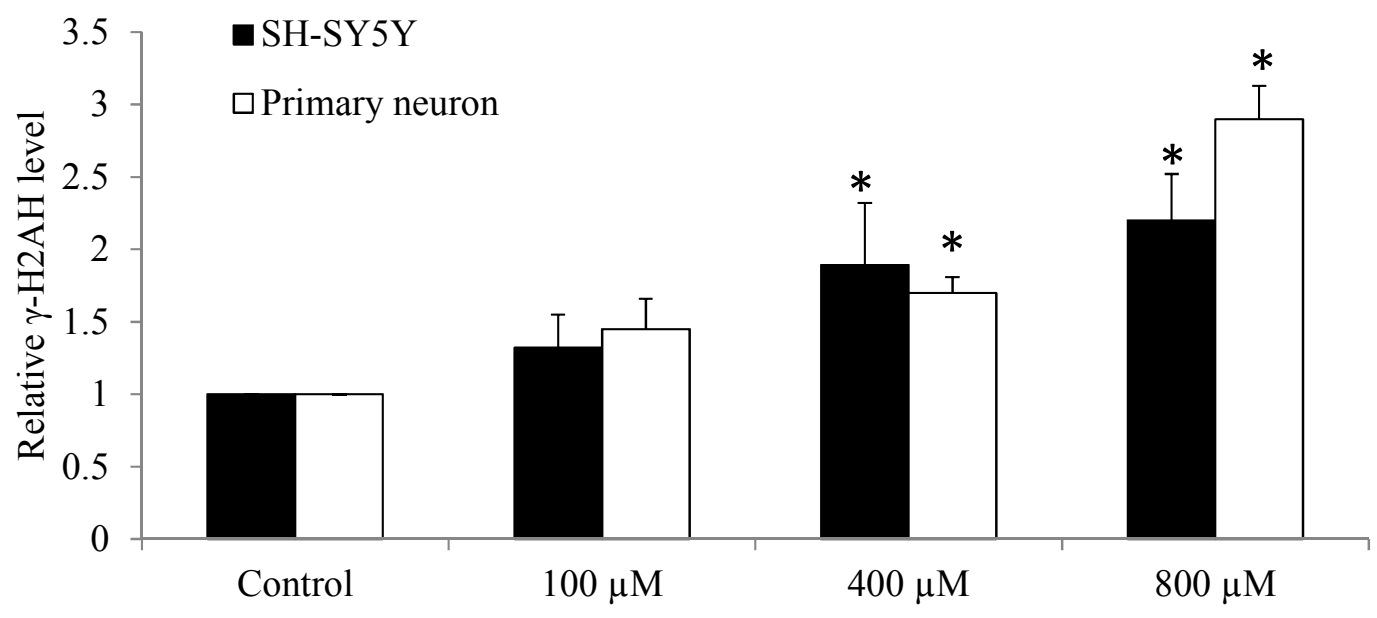

Supplementary Fig. S2. Quantification of $\boldsymbol{\gamma}$-H2AX density using image $\mathbf{J}$ software.

Piperacillin significantly increases levels of $\gamma$-H2AX in SH-SY5Y and primary neuron cells. $\gamma-$ $\mathrm{H} 2 \mathrm{AX}$ was normalized to loading control $\beta$-actin. ${ }^{*} \mathrm{p}<0.05$, compared to control.

\section{Supplementary Fig. S3}

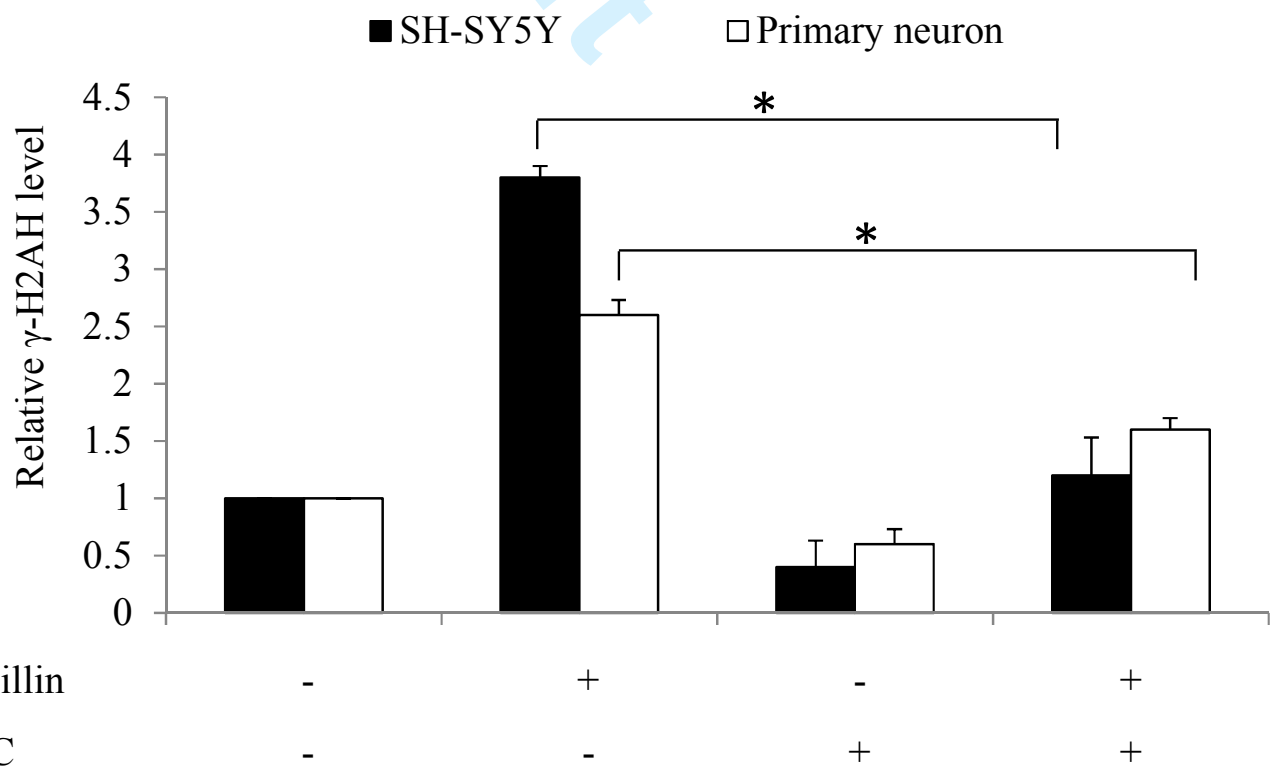

Supplementary Fig. S3. Quantification of $\gamma$-H2AX density using image $\mathbf{J}$ software. NAC reverse the effects of piperacillin in increasing $\gamma-\mathrm{H} 2 \mathrm{AH}$ level. $\gamma-\mathrm{H} 2 \mathrm{AX}$ was normalized to loading control $\beta$-actin. ${ }^{*} \mathrm{p}<0.05$, compared to piperacillin treatment alone. 